results in predominant C3 without significant Ig deposition on immunofluorescence microscopy and characteristic sausageshaped, wavy deposits by electron microscopy within the glomerulus. Disease clinically presents as proteinuria, hematuria, acute nephritis or nephrotic syndrome and acute kidney failure.

We are presenting an 8 years old boy that was brought to the clinic because of macroscopic hematuria. At initial presentation he had high blood pressure and periorbital edema. Urinalysis revealed numerous erythrocytes, proteinuria and presence of casts in the urine. Creatinine and urea concentration was elevated with low serum protein and albumin. No pleural or pericardial effusion was found. Urine culture was sterile and urinary tract ultrasound did not show anatomical anomalies or urolithiasis. He had proteinuria of $1,69 \mathrm{~g} / 24 \mathrm{~h}$ $(74,2 \mathrm{mg} / \mathrm{m} 2 / \mathrm{h})$ and albuminuria of $1096 \mathrm{mg} / 24 \mathrm{~h}$. Glomerular hematuria was confirmed with the finding of $100 \%$ dysmorphic erythrocytes in the urinary sediment. Serum C3 concentration was low with normal C4. In accordance with anamnestic data on febrile sore throat 2 weeks prior to hospitalization, the antistreptolysin titre was slightly elevated. Immunological screening excluded lupus nephritis (negative ANA and ANCA), and serological examinations excluded hepatitis $\mathrm{C}$ and $\mathrm{B}$. The patient was treated with oral antimicrobial course, fursemide and amlodipine, regulated diet. Repeated serum creatinine and urea concentrations were being improved and lastly were normalized during outpatient monitoring. Constant recovery of proteinuria and albuminuria was being observed and microscopic hematuria resolved after 4 months. Low C3 concentration persisted for another 2 months. Renal biopsy revealed focal endocapillary proliferative glomerulonephritis (GN) and GN with the dominant C3 finding on the light and immunofluorescence microscopy that may suits postinfective GN as well as C3 glomerulopathy. Electron microscopy showed a finding which is in accordance with DDD. Additional testing revealed decreased classical (43 CH50/ml; ref. range $48-103 \mathrm{CH} 50 / \mathrm{ml})$ and deficient alternative $(3 \%$; ref. range $70-125 \%$ ) pathway activity as well as slightly positive $\mathrm{C} 3 \mathrm{NeF}$, supporting the diagnosis of DDD. Genetic analysis revealed a polymorphism of the complement factor $\mathrm{H}$ gene with an increased risk of developing DDD. The child was fully recovered after supportive therapy. After a follow-up period of 2 years patient remained asymptomatic with normal kidney function. Given the poor prognosis of the disease, the proper approach to such specific glomerulopathy is important to avoid or at least to slow the progression to end-stage renal disease.

\section{TOXIC ALVEOLAR CELL INJURY IN YOUNG SMOKER}

${ }^{1}$ Anita Đula*, ${ }^{2}$ Roberta Šarkanji-Golub. ${ }^{1}$ Dom zdravlja Zagreb - Zapad; ${ }^{2}$ Irma Golmajer Vlahović, Ivan Pavić

\subsection{6/archdischild-2021-europaediatrics.495}

Introduction Almost 2,500 children under the age of 18 burn their first cigarette every day, more than 400 of them will become active smokers (1), and half will die from the effects of smoking (2). Nicotine from cigarette smoke, as well as marijuana smoke (THC), consists of toxic mixtures of gases and particulates known to have adverse effects on the lungs $(3,4)$. In this case report, we present a 15 -year-old young man with hemoptysis caused by toxic alveolar cell injury resulting from overexposure to tobacco smoke in combination with marijuana consumption.

Case Report A young man, aged 15, is admitted to our clinic due to occasional bloody cough during last couple of months. He was without known previous diseases, has been properly vaccinated and has not been in contact with tuberculosis (TB). $\mathrm{He}$ is an active athlete, did not lose weight, was not febrile lately.

Also, he is active smoker (up to 5 cig/day). At admission he was at good general condition with physical status without any abnormalities, including neat BCG scar. The laboratory work and chest radiography that was done didn't show any significant abnormalities. Additional exams (allergological, pulmonary, immunological, rheumatological, ENT) were uninformative too. Since the diagnostic work-up did not find the cause of hemoptysis, bronchoscopy was performed showing no specific macroscopic finding. Finally, a bronchoalveolar lavage (BAL) that was taken for cytologic analysis revealed toxic alveolar cell injury causing alveolar hemorrhage that was clinically manifested as hemoptysis. When confronted with the findings, the patient subsequently told us that he had been smoking excessively ( $>40$ cig/day plus occasionaly smoked marijuana) two weeks before the onset of symptoms, leading us to conclude that the problems were most likely caused by smoking a combination of tobacco and marijuana. The patient was advised to strictly avoid both active and passive smoking after which hemoptysis did not recur, which was an indirect confirmation of our assumption that smoking was a major cause of toxic alverolar cell injury.

Conclusion In addition to the most commonly known causes of hemoptysis in children with respiratory infections, aspirated foreign bodies and bronchiectasis, other rare causes should be kept in mind. To confirm toxic alveolar damage, the method of choice is bronchoscopy with cytological analysis of bronchoalveolar lavage (BAL). Also, young people need to be educated and informed about the dangers of smoking and to draw attention to the consequences of smoking.

\section{WHEN THE RESULT OF ANCILLARY TEST DOES NOT SUPPORT NEUROLOGICAL CRITERIA OF BRAIN DEATH - A CASE OF A 3-YEAR OLD GIRL WITH HEAD TRAUMA}

Nikolina Hamidović, Anamarija Čondor, Igor Berecki. University Hospital of Osijek

\subsection{6/archdischild-2021-europaediatrics.496}

Brain death occurs when a person has an irreversible brain injury causing total cessation of cerebral and brainstem function, different from coma or persistent vegetative state. The diagnosis of brain death is made clinically with different practises for need of confirmation with ancillary test in different countries.

Here we present a case of a 3-year old girl that was admitted to the emergency room after a fall with a Glasgow coma scale score 3 but still breathing spontaneously. She underwent head and cervical spine computed tomography (CT) that showed smaller bilateral frontotemporoparietal subdural hematoma and subarachnoid haemorrhage. After a neurosurgical consultation she was initially treated conservatively. About three hours later her condition profusely deteriorated with apnoea, hemodynamic instability and profound anaemia. Second CT scan showed oedema and decompression craniotomy was indicated. 72 hours after the trauma the patient was 
hemodynamically stabile and it was decided to discontinue sedation in order to evaluate brain function. 24 hours after the complete cessation of sedation there was no neurological improvement indicating she was probably brain dead. Two neurological examinations were done with observation period of 12 hours, including apnoea test, demonstrating absent cerebral and brainstem function and confirming the diagnosis of brain death. Croatian legal regulations mandate the use of confirmatory tests to supplement the clinical examination so we decided to do scintigraphic blood flow study, ancillary test of choice in our institution. First test failed to show 'hollow skull phenomenon', as the second one done 24 hours later. Because of craniotomy, it was impossible to consider the use of electroencephalography and there was not enough experience and expertise with somatosensory evoked potentials, leaving as with only blood flow studies. Since experts in their field did not expect different results with cerebral angiography or Transcranial Doppler, we repeated cerebral perfusion scintigraphy four more times in the next forty days without confirming the clinical diagnosis of brain death.

During that time there was no change in neurological status. We were able to maintain vital functions for two months in total after which the child died.

Our experience shows that even though neurological examinations demonstrated absent cerebral and brainstem function blood flow study failed to confirm the diagnosis of brain death, probably because of breached cranial vault, in this case because of craniotomy.

\section{THE CASE OF GROWTH ARREST WHICH APPEARED TO BE HYPOTHYROIDISM AND PITUATRY HYPERPLASIA}

Iryna Artcukevich*, O Zenevich, V Zhemoytiak, L Collins. Children Regional Hospial Grodno Belarus

\subsection{6/archdischild-2021-europaediatrics.497}

Background Normal growth is the main of all fundamental characteristics of childhood. Deviation from a normal pattern of growth plate is dependent on variety of different factors, including nonendocrine disorders and hormonal stimuli: growth hormone, IGF-I, sex steroids, thyroid hormones, paracrine growth factors, and cytokines.

Case We present 12 years old girl who was referred to endocrinology department (she had reduced of the height velocity in the last 3 years old and appeared overweight. The girl was born by the first normal pregnancy.

Her birth weight was $3.8 \mathrm{~kg}$, length $54 \mathrm{~cm}$. Her intellectual development and school performance was good. She did not have any complains. Her weight was $32 \mathrm{~kg}$ (10-25 cen-

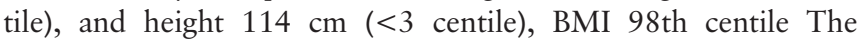
skin was dry and pale. The test showed TSH $-820 \mathrm{mIU} / \mathrm{L}$, T4f - $1.51 \mathrm{mIU} / \mathrm{L}$, and elevated antithyroperoxidase antibodies level. The US of the thyroid gland showed the reduced of the echogenic structure and multiple heterogeneous structures, confirming The Primary Hypothyroidism due to Autoimmune thyroiditis. MRI head reported The Pituitary Hyperplasia/Pituitary Adenoma secondary to The Primary Hypothyroidism. She was commenced on levothyroxine $50 \mu \mathrm{g} / \mathrm{kg}$ once daily. The repeated test after 10 days of treatment showed TSH 448.4 $\mathrm{mIU} / \mathrm{L}$ T3 $2.49 \mathrm{mIU} / \mathrm{L}$, T4 $7.15 \mathrm{mIU} / \mathrm{L}$. She was discharged home on levothyroxine and regular monitoring of TSH.
Conclusion Thyroid hormone is a major contributor to postnatal growth and hypothyroidism occurring postnatally can result in profound growth failure and arrest of skeletal maturation. The danger of chronic autoimmune thyroiditis disease lies in the fact that the disease is almost asymptomatic. Pituitary hyperplasia usually developed due to hypothyroidism, that responds well to thyroid hormone replacement therapy.

\section{THE ROLE OF INTERACTION BETWEEN HOSPITALS IN HEALTH SYSTEM}

Ani Asatryan*, E Harutyunyan, L Shahumyan, A Aghajanyan, H Shahumyan, S Khachatryan, I Atoyan, A Sahakyan, G Manukyan, K Esoyan, S Mirabyan, A Sahakyan, M Grigoryan, B Baveyan, V Asoyan, H Apresyan. Yerevan State Medical University

\subsection{6/archdischild-2021-europaediatrics.498}

The aim of our research was to investigate the diseases of 0 18 year old patients admitted to the 'Muratsan' University hospital complex (UHC) Intensive care unit (ICU) and toxicology for the last 7 years, including cases that have been reported by the 'Muratsan' UHC (ICU) from the hospitals in Yerevan and provinces and other departments of 'Muratsan' UHC.

A retrospective study was performed using medical charts up to 7 years(2012-2018) from 'Muratsan' UHC ICU and toxicology. The overall number of patients was 5152, among them 3074 were male and 2078 were female. This study included cases that have been reported by the 'Muratsan' UHC ICU and toxicology from the hospitals in Yerevan and provinces and other departments of 'Muratsan' UHC. The overall number of patients who were referred from the hospitals of Yerevan is $62(1.2 \%)$. 20(32.3\%)-Acute respiratory infection(ARI), 17(27.4\%)-Acute Intoxications(AI), 18(29\%)-Acute Drug Intoxications(ADI) and 1(1.6\%)-allergic disorders, 6 (9.7\%)-other disorders*. The referred patients were divided into 4 age groups $(0-1,1-7,7-14,14-18)$, the first group-13, the second-37, the third-2 and the fourth- 10 cases. 25 (40.3\%)-moderate, 35(56.5\%)-severe and 2(3.2\%)-extremely severe cases.

681 patients $(13.2 \%)$ were referred from regional hospitals, 38(5.6\%) patients had ARI, 400(58.7\%)-AI, 174(25.6\%)-ADI and 22(3.2\%)-allergic disorders, 47(6.9\%)-other disorders. The first age group included-72, the second-500, the third-79 and the fourth-30 cases. 334(49\%)-moderate, 345(50.7\%)-severe cases, 2(0.3\%)-extremely severe cases.

The number of internal directions was 157(3\%): 92 (58.6\%)-ARI, 7(4.5\%)-AI, 6(3.8\%)-ADI and 4(2.5\%)-allergic disorders, 48(30.6\%)-other disorders. The first age group included-26, the second-116, the third-10 and the fourth-5 cases. $11(7 \%)$ were moderate, $139(88.5 \%)$-severe and $7(4.5 \%)$ extremely severe cases.

*Diabetes mellitus, epilepsy, cancer, hematological disorders, etc.

We came to the conclusion that most cases of severe intoxication among children aged 1 to 7 years were transferred from different hospitals in Yerevan, regions and other departments of 'Muratsan' UHC. From this we can conclude that as a result of the organized work of the primary rings, patients are sent to the necessary specialized department. 